\title{
Archaeal S-layer glycoproteins: post-translational modification in the face of extremes
}

\author{
Lina Kandiba and Jerry Eichler * \\ Department of Life Sciences, Ben Gurion University of the Negev, Beersheva, Israel
}

\section{Edited by:}

Sonja-Verena Albers, University of

Freiburg, Germany

Reviewed by:

Kelly Bidle, Rider University, USA

Reinhard Rachel, University of

Regensburg, Germany

*Correspondence:

Jerry Eichler, Department of Life Sciences, Ben Gurion University of the Negev, PO Box 653, Beersheva 84105, Israel

e-mail: jeichler@bgu.ac.il
Corresponding to the sole or basic component of the surface (S)-layer surrounding the archaeal cell in most known cases, S-layer glycoproteins are in direct contact with the harsh environments that characterize niches where Archaea can thrive. Accordingly, early work examining archaeal S-layer glycoproteins focused on identifying those properties that allow members of this group of proteins to maintain their structural integrity in the face of extremes of temperature, $\mathrm{pH}$, and salinity, as well as other physical challenges. However, with expansion of the list of archaeal strains serving as model systems, as well as growth in the number of molecular tools available for the manipulation of these strains, studies on archaeal S-layer glycoproteins are currently more likely to consider the various post-translational modifications these polypeptides undergo. For instance, archaeal S-layer glycoproteins can undergo proteolytic cleavage, both $\mathrm{N}$ - and $\mathrm{O}$-glycosylation, lipidmodification and oligomerization. In this mini-review, recent findings related to the posttranslational modification of archaeal S-layer glycoproteins are considered.

Keywords: Archaea, lipid modification, post-translational modification, protein glycosylation, S-layer glycoprotein
Although Archaea are now recognized as denizens of an enormous range of environments, they remain best known in their capacities as extremophiles, namely organisms able to thrive in some of the most physically challenging settings on the planet. In direct contact with these often hostile surroundings, the archaeal cell surface must not only maintain its integrity but also must carry out a variety of normal physiological functions. In Bacteria, the cell boundary consists of membranes and a peptidoglycan-based cell wall together with other polysaccharidebased molecules (e.g., lipopolysaccharide, teichoic acid) and proteins (Braun, 1975; Lugtenberg and Van Alphen, 1983; Raetz et al., 2007), in many cases comprising a surface (S)-layer (Fagan and Fairweather, 2014). By contrast, the cell wall in Archaea tends to be much simpler. Apart from a number of documented examples (König, 2001), the S-layer, in many cases comprising a single protein species but not always (Peters et al., 1995; Grogan, 1996; Veith et al., 2009), corresponds to the sole cell wall structure (Eichler, 2003; Albers and Meyer, 2011). Studies from several groups studying different Archaea have shown that the S-layer glycoprotein is not just a standardized building block used to generate the two-dimensional lattice of the S-layer but rather that S-layer glycoproteins undergo a variety of posttranslational modifications. In this mini-review, recent findings concerning such processing of archaeal S-layer glycoproteins are considered.

\section{DIFFERENCES IN THE SUGAR COATING}

The S-layer glycoprotein of the haloarchaeon Halobacterium salinarum offered the first example of $N$-glycosylation in a domain other than the Eukarya (Mescher and Strominger, 1976a). This observation led to a flurry of biochemical activity aimed at describing the composition of $\mathrm{N}$-linked glycans decorating the Hbt. salinarum S-layer glycoprotein and their biosynthesis (cf. Lechner and Wieland, 1989). However, the lack of sufficient genetic tools for manipulating this and other archaeal species shown to contain glycosylated S-layer proteins (Sumper et al., 1990; Brockl et al., 1991; Karcher et al., 1993) stood in the way of gaining detailed information into such post-translational modification of this protein. Since then, the sequencing of a growing list of archaeal genomes, the development of techniques for manipulating the genetic content of numerous strains and the analytical power of mass spectrometry have been combined to help clear obstacles encountered by earlier studies of S-layer glycoprotein $N$-glycosylation.

Genomic analyses point both to the presence of S-layer glycoproteins and $\mathrm{N}$-glycosylation machineries in almost all sequenced Archaea (Magidovich and Eichler, 2009; Albers and Meyer, 2011; Kaminski et al., 2013a). Still, the majority of research on archaeal S-layer glycoprotein $\mathrm{N}$-glycosylation to date has focused on Methanococcus voltae, Methanococcus maripaludis, Sulfolobus acidocaldarius, and Haloferax volcanii (for recent review, see Jarrell et al., 2014). In each of these species, genes involved in the assembly and attachment of N-linked glycans and often their protein products have been studied. Yet, apart from $S$. acidocaldarius, where $N$-glycosylation is essential for cell survival (Meyer and Albers, 2014), the elimination of such protein processing seemingly has limited impact on the organism (Abu-Qarn and Eichler, 2006; Chaban et al., 2006; VanDyke et al., 2009). As such, one can ask why Archaea devote such a significant number of genes to this post-translational modification. Recent studies on 
Hfx. volcanii S-layer glycoprotein have begun to shed light on this point.

The $H f x$. volcanii S-layer glycoprotein contains seven putative $N$-glycosylation sites (Sumper et al., 1990). Of these, Asn-13 and Asn-83 are modified by a pentasaccharide comprising a hexose, two hexuronic acids, a methyl ester of hexuronic acid and a mannose (Abu-Qarn et al., 2007; Guan et al., 2010; Magidovich et al., 2010). However, when $H f x$. volcanii cells are grown in medium containing $1.75 \mathrm{M} \mathrm{NaCl}$ ("low salt" conditions) rather than $3.4 \mathrm{M}$ $\mathrm{NaCl}$ ("high salt" conditions), Asn-498 is modified by a distinct glycan comprising a sulfated hexose, two hexoses and a rhamnose (Guan et al., 2012). Indeed, the same glycan had been reported earlier as bound to dolichol phosphate in $H f x$. volcanii grown in the presence of $1.25 \mathrm{M} \mathrm{NaCl}$ (Kuntz et al., 1997), the lipid carrier that serves as the platform for $N$-glycan assembly in this and other Archaea (Lechner et al., 1985; Guan et al., 2010; Calo et al., 2011). As such, it would appear that the $H f x$. volcanii S-layer glycoprotein undergoes differential $N$-glycosylation as a function of environmental salinity. While it remains to be defined how such differential S-layer glycoprotein $N$-glycosylation translates into an appropriate response to changes in surrounding salt levels, the path involved in the biogenesis of the so-called "low-salt" tetrasaccharide has been revealed (Kaminski et al., 2013b). Unexpectedly, the cluster of genes involved does not include an obvious oligosaccharyltransferase, namely that enzyme responsible for transferring a glycan from its lipid carrier to select Asn residues of target proteins (Mohorko et al., 2011). The observation that AglB, the only known archaeal oligosaccharyltransferase (AbuQarn and Eichler, 2006; Chaban et al., 2006), is not involved in "low-salt" tetrasaccharide attachment implies the existence of a novel yet undefined enzyme as serving this role (Kaminski et al., 2013b).

It is possible that $N$-glycosylation of the $H f x$. volcanii S-layer glycoprotein is even more complicated still. It was recently reported that the Asn-732 position is modified by a sulfoquinovose-hexose-based glycan, $N$-linked via a chitobiose core (Parente et al., 2014). Moreover, the composition of this glycan was modified in response to the absence or presence of a membrane-localized rhomboid protease. The presence of such a glycan in $H f x$. volcanii is surprising, given this organism does not contain a homolog of S. acidocaldarius Agl3 (Meyer et al., 2011), a UDP-sulfoquinovose synthase responsible for converting UDP-glucose and sodium sulfite into UDP-sulfoquinovose, the activated form of this sugar that is presumably used in $S$. acidocaldarius and presumably $H f x$. volcanii $N$-glycosylation. It should also be noted that Asn-732 is found in the same Cterminal region as a cluster of $O$-glycosylated threonine residues (Sumper et al., 1990) and a lipid anchor (see below). This suggests that post-translational modification of the Hfx. volcanii S-layer glycoprotein C-terminal region is a complex event that requires the orchestrated involvement of numerous protein processing pathways.

Unlike $H f x$. volcanii, which must cope with an environment characterized by molar concentrations of salt, S. acidocaldarius is a thermophile that grows optimally at $75-80^{\circ} \mathrm{C}$ and $\mathrm{pH} 2-$ 3 (Brock et al., 1972). Possibly due to the challenges presented by its surroundings, not only is $N$-glycosylation essential in
S. acidocaldarius (Meyer and Albers, 2014) but at least one of the glycoproteins comprising the S-layer in this species (SlaA) presents an extremely high number of $N$-glycosylation sites. The 1,395 amino acid-long protein contains 31 potential sites for $N$-glycosylation scattered throughout the polypeptide, translating to an $N$-glycosylation site every 45 residues on average, with the highest densely of such sites being seen in the Cterminal quarter of the protein (Peyfoon et al., 2010). In the region spanning Lys-1004 to Gln-1395, nine of the 11 potential $N$-glycosylation sites were experimentally verified as being charged with a tri-branched hexasaccharide comprising a glucose, a mannose, two $\mathrm{N}$-acetylglucosamines and a sulfoquinovose, an unusual sugar routinely found in chloroplasts and photosynthetic bacteria (Zahringer et al., 2000; Peyfoon et al., 2010). Indeed, a tallying of the number of putative $N$-glycosylation sites in 20 different archaeal S-layer glycoproteins reveals that the S-layer glycoproteins of thermo(acido)philes can contain up to 20-fold more such sites than do S-layer glycoproteins in species isolated from other growth conditions (Jarrell et al., 2014). Based on this comparison, it was proposed that such high densities of $\mathrm{N}$ glycosylation sites reflect the need for a rigid and stable cell wall to cope with the challenges of elevated temperatures and acidity encountered by thermo(acido)philic Archaea.

The importance of S-layer glycoprotein glycosylation was also demonstrated in recent work linking the activity of a transcription factor controlling the expression of genes involved in sugar metabolism with S-layer glycoprotein glycosylation and hence, with the maintenance of cell shape in Hbt. salinarum (Todor et al., 2014). TrmB binds to the promoters of over 110 genes encoding products involved in various metabolic processes in response to glucose concentrations. Yet, Hbt. salinarum does not catabolize glucose, cannot use glucose as the sole carbon or energy source and does not actively transport glucose from the media (Gochnauer and Kushner, 1969; Severina et al., 1990). As such, it was proposed that TrmB activity ensures that sufficient amounts of glucose and other monosaccharides are available for S-layer glycoprotein glycosylation. S-layer glycoprotein glycosylation is directly related to Hbt. salinarum maintaining its rod-like shape, with a loss of $N$-glycosylation leading to the appearance of round cells (Mescher and Strominger, 1976b). Hence, TrmB activity is linked to Hbt. salinarum shape and by extension to cell growth, since this process requires the presence of sufficient fully processed S-layer glycoprotein.

Finally, O-glycosylation, where the glycan is linked to the hydroxyl group of Ser or Thr residues, has been reported for both the Hbt. salinarum and the Hfx. volcanii S-layer glycoproteins (Mescher and Strominger, 1976a; Sumper et al., 1990). In both proteins, Thr-rich regions adjacent to the predicted membranespanning domain of the protein are modified with galactoseglucose disaccharides. Still today, nothing is known of the pathways responsible for $\mathrm{O}$-glycosylation in Archaea.

\section{HANGING ON BY A LIPID}

Just as S-layer glycoproteins have served as tractable reporters of archaeal protein glycosylation, they have also been central to our understanding of lipid modification in Archaea, namely the covalent linkage of lipid-based groups to a polypeptide chain. 


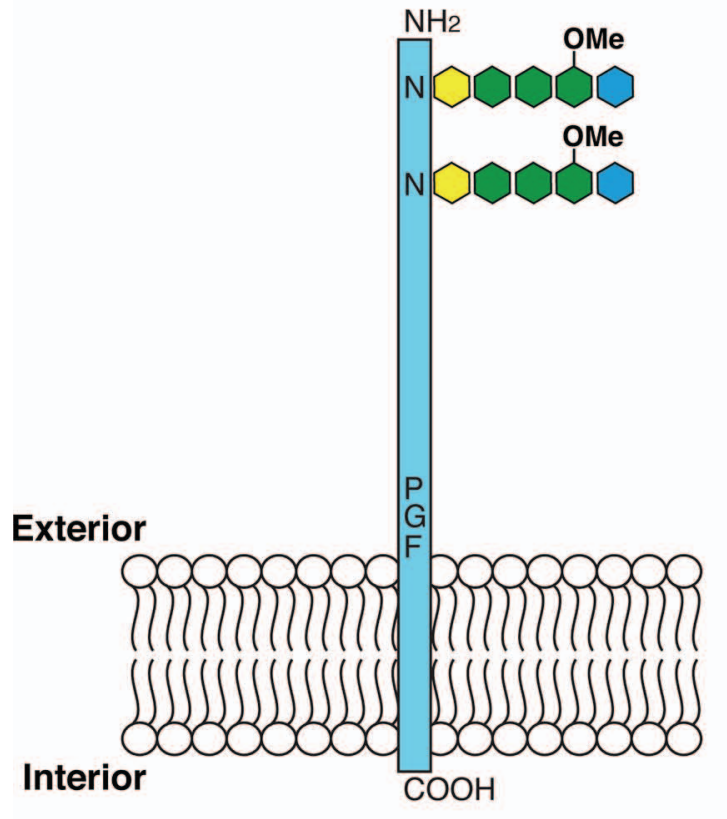

ArtA

FIGURE 1 | Schematic depiction of the proposed Hfx. volcanii S-layer glycoprotein lipid modification process. In Hfx. volcanii, the S-layer glycoprotein is synthesized with a C-terminal membrane-spanning domain. ArtA cleaves the protein at a PGF motif immediately upstream of the transmembrane domain. The cleaved S-layer glycoprotein fragment is transferred to a waiting archaetidic acid anchor, schematically depicted in purple (detailed structure provided). Alternatively, attachment of the lipid anchor could proceed protein
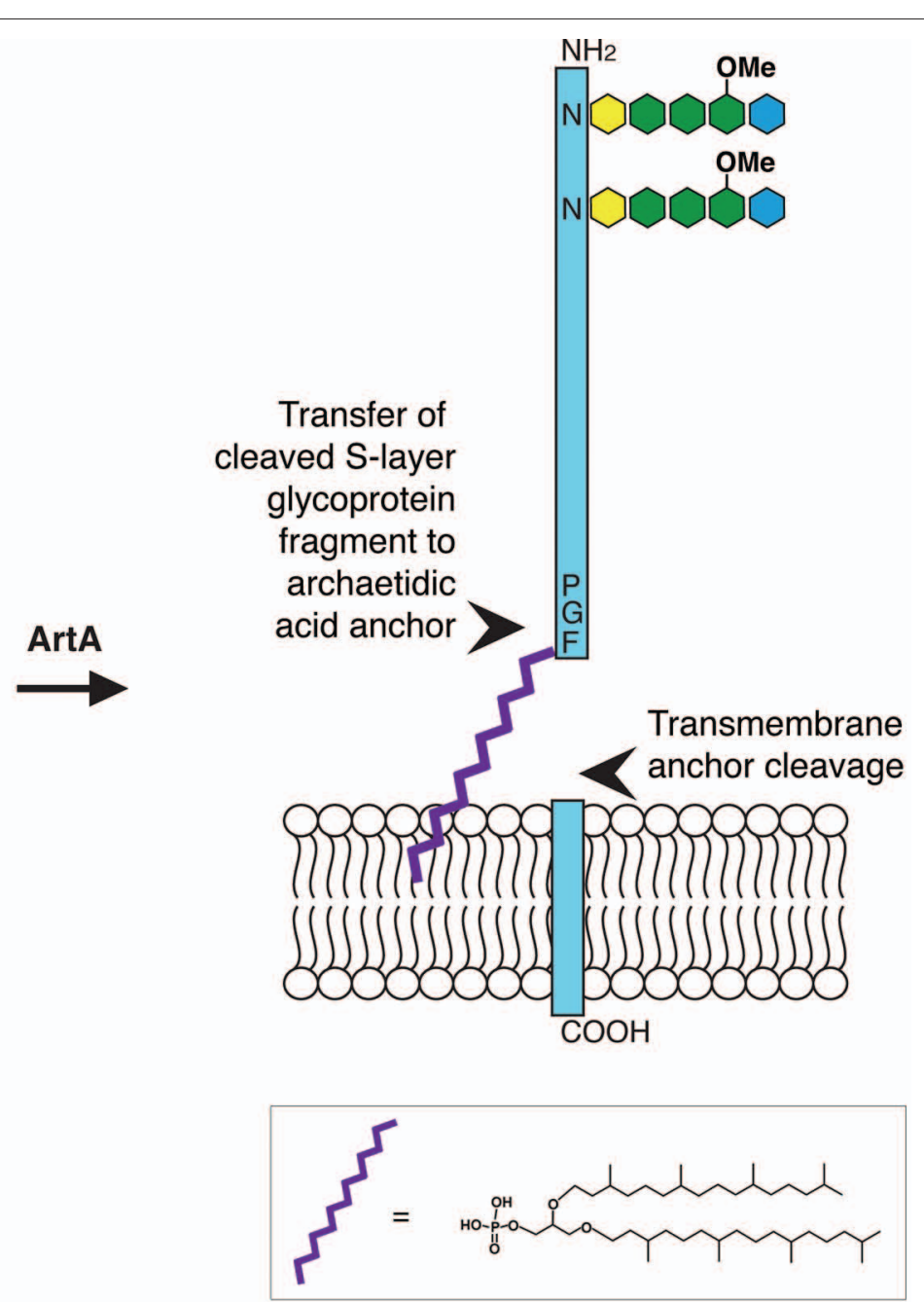

cleavage. In either case, two S-layer glycoprotein populations appear. Such lipid modification transpires following N-glycosylation of the protein. Of the seven putative N-glycosylation sites, Asn-13 and Asn-83 are modified by a pentasaccharide comprising a hexose (yellow), three hexuronic acids (green; the last a methyl ester of hexuronic acid) and a mannose (blue). The temporal relation between lipid modification and O-glycosylation of a cluster of Thr residues found above the cleavage site (not shown) remains to be determined.
Relying on various biochemical approaches, it was shown that the S-layer glycoproteins of Hbt. salinarum and $H f x$. volcanii undergo lipid modification (Kikuchi et al., 1999; Konrad and Eichler, 2002). However, it is only of late that insight into the process of such lipid modification has been provided.

Analysis of the deduced amino acid sequence of the $H f x$. volcanii S-layer glycoprotein (Sumper et al., 1990) predicts the existence of a 20-residue-long C-terminal membrane-spanning domain, thought to anchor the protein within the membrane. At the same time, it was shown that EDTA treatment leads to the release of the S-layer glycoprotein into the surrounding growth medium (Cline et al., 1989). Solving the paradox of how an apparently integral membrane protein could be solubilized by divalent cation chelation began with studies showing incorporation of radiolabeled polyprenol precursors into the $H f x$. volcanii S-layer glycoprotein. This observation led to the conclusion that the protein is subjected to magnesium-dependent processing associated with lipid modification (Eichler, 2001; Konrad and Eichler, 2002). A decade later, a combination of sequential solubilization steps, native gel electrophoresis and mass spectrometry pointed to the existence of two distinct subpopulations of the S-layer glycoprotein, the first corresponding to an EDTA-solubilized pool anchored to the membrane via a covalently linked archaetidic acid lipid anchor and the second representing detergent-solubilized pool anchored to the membrane likely via the $\mathrm{C}$-terminal membrane-spanning domain 
(Kandiba et al., 2013). Both S-layer glycoproteins were shown to be $N$-glycosylated.

In the same period, it was proposed that the Pro-Gly-Phe motif found just upstream of the presumed C-terminal membranespanning domain of the Hfx. volcanii and Hbt. salinarum S-layer glycoproteins is processed similarly as a comparable motif found in certain membrane-linked Gram-positive bacterial proteins (Haft et al., 2012). In Bacteria, this motif is cleaved by a transpeptidase called an exosortase and the released protein is linked to the cell wall via a waiting lipid anchor. Accordingly, genome sequence analysis predicted the existence of an archaeal version of exosortase, termed archaeosortase A (ArtA). Subsequent genetic and biochemical work confirmed not only the existence of ArtA but also its ability to cleave the $H f x$. volcanii S-layer glycoprotein at the C-terminal Pro-Gly-Phe motif described above (Abdul Halim et al., 2013).

Together, the results of these recent studies argue that in $H f x$. volcanii (and likely in Hbt. salinarum as well), the S-layer glycoprotein is initially synthesized with a C-terminal membranespanning domain. This precursor is cleaved by ArtA and the processed S-layer glycoprotein is transferred to a waiting archaetidic acid anchor lipid anchor in a magnesium-dependent manner. Still, as only selected aspects of this hypothesized pathway (Figure 1) have been demonstrated, further experiments await.

\section{CONCLUSION}

Corresponding to the building block of the S-layer, the outermost limit of the archaeal cell surface, S-layer glycoproteins are not only in direct contact with the harsh environments Archaea can inhabit but are also amongst the first archaeal proteins to encounter any changes in those environments. Post-translational modification of S-layer glycoproteins offer a rapid and reversible response to such changes. Soon, ongoing efforts in laboratories around the world will not only provide further insight into the pathways recruited for these protein processing events but will also hopefully reveal how such modifications affect S-layer structure and stability. Indeed, with the availability of high resolution structures of archaeal S-glycoproteins (Arbing et al., 2012), it will be possible to obtain detailed understanding of the contributions of posttranslational modification to S-layer architecture not only as a function of environment but also of growth stage and other physiological conditions.

\section{AUTHOR CONTRIBUTIONS}

All authors made substantial contributions to the acquisition, analysis, and interpretation of data described in this report. All authors critically reviewed the report and approved the final version. All authors agree to be accountable for all aspects of the work in ensuring that questions related to the accuracy or integrity of any part of the work are appropriately investigated and resolved.

\section{ACKNOWLEDGMENTS}

Research in the Eichler laboratory is supported by the Israel Science Foundation (grant 8/11) and the US Army Research Office (W911NF-11-1-520).

\section{REFERENCES}

Abdul Halim, M. F., Pfeiffer, F., Zou, J., Frisch, A., Haft, D., Wu, S., et al. (2013). Haloferax volcanii archaeosortase is required for motility, mating, and Cterminal processing of the S-layer glycoprotein. Mol. Microbiol. 88, 1164-1175. doi: $10.1111 / \mathrm{mmi} .12248$

Abu-Qarn, M., and Eichler, J. (2006). Protein N-glycosylation in Archaea: defining Haloferax volcanii genes involved in S-layer glycoprotein glycosylation. Mol. Microbiol. 61, 511-525. doi: 10.1111/j.1365-2958.2006.05252.x

Abu-Qarn, M., Yurist-Doutsch, S., Giordano, A., Trauner, A., Morris, H. R., Hitchen, P., et al. (2007). Haloferax volcanii AglB and AglD are involved in Nglycosylation of the S-layer glycoprotein and proper assembly of the surface layer. J. Mol. Biol. 374, 1224-1236. doi: 10.1016/j.jmb.2007.10.042

Albers, S. V., and Meyer, B. H. (2011). The archaeal cell envelope. Nat. Rev. Microbiol. 9, 414-426. doi: 10.1038/nrmicro2576

Arbing, M. A., Chan, S., Shin, A., Phan, T., Ahn, C. J., Rohlin, L., et al. (2012). Structure of the surface layer of the methanogenic archaean Methanosarcina acetivorans. Proc. Natl. Acad. Sci. U.S.A. 109, 18112-18117. doi: 10.1073/pnas. 1120595109

Braun, V. (1975). Covalent lipoprotein from the outer membrane of Escherichia coli. Biochim. Biophys. Acta 415, 335-77. doi: 10.1016/0304-4157(75)90013-1

Brock, T. D., Brock, K. M., Belly, R. T., and Weiss, R. L. (1972). Sulfolobus: a new genus of sulfur-oxidizing bacteria living at low $\mathrm{pH}$ and high temperature. Arch. Microbiol. 84, 54-68.

Brockl, G., Behr, M., Fabry, S., Hensel, R., Kaudewitz, H., Biendl, E., et al. (1991). Analysis and nucleotide sequence of the genes encoding the surface-layer glycoproteins of the hyperthermophilic methanogens Methanothermus fervidus and Methanothermus sociabilis. Eur. J. Biochem. 199, 147-152. doi: 10.1111/j.14321033.1991.tb16102.x

Calo, D., Guan, Z., Naparstek, S., and Eichler, J. (2011). Different routes to the same ending: comparing the $N$-glycosylation processes of Haloferax volcanii and Haloarcula marismortui, two halophilic Archaea from the Dead Sea. Mol. Microbiol. 81, 1166-1177. doi: 10.1111/j.1365-2958.2011.07781.x

Chaban, B., Voisin, S., Kelly, J., Logan, S. M., and Jarrell, K. F. (2006). Identification of genes involved in the biosynthesis and attachment of Methanococcus voltae $\mathrm{N}$-linked glycans: insight into $\mathrm{N}$-linked glycosylation pathways in Archaea. Mol. Microbiol. 61, 259-268. doi: 10.1111/j.1365-2958.2006.05226.x

Cline, S. W., Lam, W. L., Charlebois, R. L., Schalkwyk, L. C., and Doolittle, W. F. (1989). Transformation methods for halophilic archaebacterial. Can. J. Microbiol. 35, 148-152. doi: 10.1139/m89-022

Eichler, J. (2001). Post-translational modification unrelated to protein glycosylation follows translocation of the S-layer glycoprotein across the plasma membrane of the haloarchaeon Haloferax volcanii. Eur. J. Biochem. 268, 4366-4373. doi: 10.1046/j.1432-1327.2001.02361.x

Eichler, J. (2003). Facing extremes: archaeal surface-layer (glyco-) proteins. Microbiology 149, 3347-3351. doi: 10.1099/mic.0.26591-0

Fagan, R. P., and Fairweather, N. F. (2014). Biogenesis and functions of bacterial S-layers. Nat. Rev. Microbiol. 12, 211-222. doi: 10.1038/nrmicro3213

Gochnauer, M. B., and Kushner, D. J. (1969). Growth and nutrition of extremely halophilic bacteria. Can. J. Microbiol. 15, 1157-1165. doi: 10.1139/ m69-211

Grogan, D. W. (1996). Organization and interactions of cell envelope proteins of the extreme thermoacidophile Sulfolobus acidocaldarius. Can. J. Microbiol. 42, 1163-1171. doi: 10.1139/m96-148

Guan, Z., Naparstek, S., Calo, D., and Eichler, J. (2012). Protein glycosylation as an adaptive response in Archaea: growth at different salt concentrations leads to alterations in Haloferax volcanii S-layer glycoprotein $N$-glycosylation. Environ. Microbiol. 14, 743-753. doi: 10.1111/j.1462-2920.2011.02625.x

Guan, Z., Naparstek, S., Kaminski, L., Konrad, Z., and Eichler, J. (2010). Distinct glycan-charged phosphodolichol carriers are required for the assembly of the pentasaccharide $\mathrm{N}$-linked to the Haloferax volcanii S-layer glycoprotein. Mol. Microbiol. 78, 1294-1303. doi: 10.1111/j.1365-2958.2010.07405.x

Haft, D. H., Payne, S. H., and Selengut, J. D. (2012). Archaeosortases and exosortases are widely distributed systems linking membrane transit with posttranslational modification. J. Bacteriol. 194, 36-48. doi: 10.1128/JB.06026-11

Jarrell, K. F., Ding, Y., Meyer, B. H., Albers, S. V., Kaminski, L., and Eichler, J. (2014). $N$-linked glycosylation in Archaea: a structural, functional, and genetic analysis. Microbiol. Mol. Biol. Rev. 78, 304-341. doi: 10.1128/MMBR.00052-13

Kaminski, L., Lurie-Weinberger, M. N., Allers, T., Gophna, U., and Eichler, J. (2013a). Phylogenetic- and genome-derived insight into the evolutionary 
history of $N$-glycosylation in Archaea. Mol. Phylogenet. Evol. 68, 327-339. doi: 10.1016/j.ympev.2013.03.024

Kaminski, L., Guan, Z., Yurist-Doutsch, S., and Eichler, J. (2013b). Two distinct $\mathrm{N}$-glycosylation pathways process the Haloferax volcanii S-layer glycoprotein upon changes in environmental salinity. mBio 4:e00716-13. doi: 10.1128/mBio. 00716-13

Kandiba, L., Guan, Z., and Eichler, J. (2013). Lipid modification gives rise to two distinct Haloferax volcanii S-layer glycoprotein populations. Biochim. Biophys. Acta 1828, 938-943. doi: 10.1016/j.bbamem.2012.11.023

Karcher, U., Schroder, H., Haslinger, E., Allmaier, G., Schreiner, R., Wieland, F., et al. (1993). Primary structure of the heterosaccharide of the surface glycoprotein of Methanothermus fervidus. J. Biol. Chem. 268, 26821-26826.

Kikuchi, A., Sagami, H., and Ogura, K. (1999). Evidence for covalent attachment of diphytanylglyceryl phosphate to the cell-surface glycoprotein of Halobacterium halobium. J. Biol. Chem. 274, 18011-18016. doi: 10.1074/jbc.274.25.18011

König, H. (2001). Archaeal cell walls. eLS. doi: 10.1038/npg.els.0000384

Konrad, Z., and Eichler, J. (2002). Lipid modification of proteins in Archaea: attachment of a mevalonic acid-based lipid moiety to the surface-layer glycoprotein of Haloferax volcanii follows protein translocation. Biochem. J. 366, 959-964. doi: 10.1042/BJ20020757

Kuntz, C., Sonnenbichler, J., Sonnenbichler, I., Sumper, M., and Zeitler, R. (1997). Isolation and characterization of dolichol-linked oligosaccharides from Haloferax volcanii. Glycobiology 7, 897-904. doi: 10.1093/glycob/7.7.897

Lechner, J., and Wieland, F. (1989). Structure and biosynthesis of prokaryotic glycoproteins. Annu. Rev. Biochem. 58, 173-194. doi: 10.1146/annurev.bi.58. 070189.001133

Lechner, J., Wieland, F., and Sumper, M. (1985). Biosynthesis of sulfated saccharides $\mathrm{N}$-glycosidically linked to the protein via glucose. Purification and identification of sulfated dolichyl monophosphoryl tetrasaccharides from halobacteria. J. Biol. Chem. 260, 860-866.

Lugtenberg, B., and Van Alphen, L. (1983). Molecular architecture and functioning of the outer membrane of Escherichia coli and other gram-negative bacteria. Biochim. Biophys. Acta 737, 51-115. doi: 10.1016/0304-4157(83)90014-X

Magidovich, H., and Eichler, J. (2009). Glycosyltransferases and oligosaccharyltransferases in Archaea: putative components of the $\mathrm{N}$-glycosylation pathway in the third domain of life. FEMS Microbiol. Lett. 300, 122-130. doi: 10.1111/j.1574-6968.2009.01775.x

Magidovich, H., Yurist-Doutsch, S., Konrad, Z., Ventura, V. V., Dell, A., Hitchen, P. G., et al. (2010). AglP is a S-adenosyl-L-methionine-dependent methyltransferase that participates in the $\mathrm{N}$-glycosylation pathway of Haloferax volcanii. Mol. Microbiol. 76, 190-199. doi: 10.1111/j.1365-2958.2010.07090.x

Mescher, M. F., and Strominger, J. L. (1976a). Purification and characterization of a prokaryotic glucoprotein from the cell envelope of Halobacterium salinarium. J. Biol. Chem. 251, 2005-2014.

Mescher, M. F., and Strominger, J. L. (1976b). Structural (shape-maintaining) role of the cell surface glycoprotein of Halobacterium salinarium. Proc. Natl. Acad. Sci. U.S.A. 73, 2687-2691. doi: 10.1073/pnas.73.8.2687

Meyer, B. H., and Albers, S. V. (2014). AglB, catalyzing the oligosaccharyl transferase step of the archaeal $N$-glycosylation process, is essential in the thermoacidophilic crenarchaeon Sulfolobus acidocaldarius. Microbiologyopen 3, 531-543. doi: $10.1002 / \mathrm{mbo} .185$

Meyer, B. H., Zolghadr, B., Peyfoon, E., Pabst, M., Panico, M., Morris, H. R., et al. (2011). Sulfoquinovose synthase-an important enzyme in the $\mathrm{N}$ glycosylation pathway of Sulfolobus acidocaldarius. Mol. Microbiol. 82, 11501163. doi: 10.1111/j.1365-2958.2011.07875.x

Mohorko, E., Glockshuber, R., and Aebi, M. (2011). Oligosaccharyltransferase: the central enzyme of $N$-linked protein glycosylation. J. Inherit. Metab. Dis. 34, 869878. doi: 10.1007/s10545-011-9337-1
Parente, J., Casabuono, A., Ferrari, M. C., Paggi, R. A., De Castro, R. E., Couto, A. S., et al. (2014). A rhomboid protease gene deletion affects a novel oligosaccharide $\mathrm{N}$-linked to the S-layer glycoprotein of Haloferax volcanii. J. Biol. Chem. 289, 11304-11317. doi: 10.1074/jbc.M113.546531

Peters, J., Nitsch, M.,Kuhlmorgen, B., Golbik, R., Lupas, A., Kellermann, J., et al. (1995). Tetrabrachion: a filamentous archaebacterial surface protein assembly of unusual structure and extreme stability. J. Mol. Biol. 245, 385-401. doi: 10.1006/jmbi.1994.0032

Peyfoon, E., Meyer, B., Hitchen, P. G., Panico, M., Morris, H. R., Haslam, S. M., et al. (2010). The S-layer glycoprotein of the crenarchaeote Sulfolobus acidocaldarius is glycosylated at multiple sites with chitobiose-linked $N$-glycans. Archaea pii: 754101. doi: 10.1155/2010/754101

Raetz, C. R., Reynolds, C. M., Trent, M. S., and Bishop, R. E. (2007). Lipid A modification systems in gram-negative bacteria. Annu. Rev. Biochem. 76, 295329. doi: 10.1146/annurev.biochem.76.010307.145803

Severina, L. O., Pimenov, N. V., and Plakunov, V. K. (1990). Glucose transport into the extremely halophilic archaebacteria. Arch. Microbiol. 155, 131-136. doi: 10.1007/BF00248606

Sumper, M., Berg, E., Mengele, R., and Strobel, I. (1990). Primary structure and glycosylation of the S-layer protein of Haloferax volcanii. J. Bacteriol. 172, 71117118.

Todor, H., Dulmage, K., Gillum, N., Bain, J. R., Muehlbauer, M. J., and Schmid, A. K. (2014). A transcription factor links growth rate and metabolism in the hypersaline adapted archaeon Halobacterium salinarum. Mol. Microbiol. 93, 1172-1182. doi: $10.1111 / \mathrm{mmi} .12726$

VanDyke, D. J., Wu, J., Logan, S. M., Kelly, J. F., Mizuno, S., Aizawa, S., et al. (2009). Identification of genes involved in the assembly and attachment of a novel flagellin $N$-linked tetrasaccharide important for motility in the archaeon Methanococcus maripaludis. Mol. Microbiol. 72, 633-644. doi: 10.1111/j.13652958.2009.06671.x

Veith, A., Klingl, A., Zolghadr, B., Lauber, K., Mentele, R., Lottspeich, F., et al. (2009). Acidianus, Sulfolobus and Metallosphaera surface layers: structure, composition and gene expression. Mol. Microbiol. 73, 58-72. doi: 10.1111/j.13652958.2009.06746.x

Zahringer, U., Moll, H., Hettmann, T., Knirel, Y. A., and Schafer, G. (2000). Cytochrome b558/566 from the archaeon Sulfolobus acidocaldarius has a unique Asn-linked highly branched hexasaccharide chain containing 6sulfoquinovose. Eur. J. Biochem. 267, 4144-4149. doi: 10.1046/j.1432-1327.2000. 01446.x

Conflict of Interest Statement: The authors declare that the research was conducted in the absence of any commercial or financial relationships that could be construed as a potential conflict of interest.

Received: 15 September 2014; accepted: 14 November 2014; published online: 26 November 2014.

Citation: Kandiba L and Eichler J (2014) Archaeal S-layer glycoproteins: posttranslational modification in the face of extremes. Front. Microbiol. 5:661. doi: 10.3389/fmicb.2014.00661

This article was submitted to Microbial Physiology and Metabolism, a section of the journal Frontiers in Microbiology.

Copyright (๑) 2014 Kandiba and Eichler. This is an open-access article distributed under the terms of the Creative Commons Attribution License (CC BY). The use, distribution or reproduction in other forums is permitted, provided the original author(s) or licensor are credited and that the original publication in this journal is cited, in accordance with accepted academic practice. No use, distribution or reproduction is permitted which does not comply with these terms. 